\title{
Preventing Obesity and Eating Disorders through Behavioural Modifications: the SPLENDID Vision
}

\author{
Christos Maramis*, Christos Diou ${ }^{\dagger}$, Ioannis Ioakeimidis ${ }^{\ddagger}$, Irini Lekka*, Gabriela Dudnik ${ }^{\S}$, \\ Monica Mars ${ }^{\Uparrow}$, Nikos Maglaveras*, Cecilia Bergh", and Anastasios Delopoulos ${ }^{\dagger}$ \\ *Department of Medicine, Aristotle University of Thessaloniki, 54124, Greece \\ ${ }^{\dagger}$ Department of Electrical \& Computer Engineering, Aristotle University of Thessaloniki, 54124, Greece \\ ${ }^{\ddagger}$ Division for Applied Neuroendocrinology, Karolinska Institutet, S-141 57, Sweden

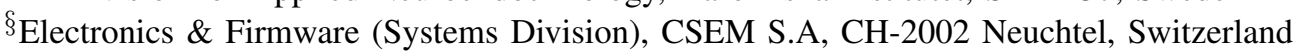 \\ IDivision of Human Nutrition, Wageningen University, PO Box 8129, NL-6700 EV, Wageningen, The Netherlands \\ $\|_{\text {Mando Group AB, S-141 57, Sweden }}$
}

\begin{abstract}
Recent intensive research in the fields of obesity and eating disorders has proved most traditional interventions inadequate: The obesity-targeting interventions have either failed or are strongly social context dependent, while the interventions for eating disorders have poor results and high levels of relapse. On the contrary, recent randomized control trials have illustrated that supervised training of patients to eat and move in a nonpathological way is effective in the prevention of both obesity and eating disorders. Applying the same kind of methodologies to the general population in real life conditions for prevention purposes comes as the logical next step. SPLENDID is a recently initiated EU-funded collaborative project that intends to develop a personalised guidance system for helping and training children and young adults to improve their eating and activity behaviour. By combining expertise in behavioural patterns with current advancements in intelligent systems and sensor technologies, SPLENDID is going to detect subjects at risk for developing obesity or eating disorders and offer them enhanced monitoring and guidance to prevent further disease progression. Both behavioural data collection and system evaluation are going to be performed via pilot studies supported by expert health professionals.
\end{abstract}

Keywords-Obesity \& eating disorders prevention, eating \& activity monitoring, behaviour modification, personalised guidance system

\section{INTRODUCTION}

Worldwide obesity has more than doubled since 1980 resulting today in more than 1.4 billion overweight and 500 million obese adults [1]. Meanwhile, the prevalence of eating disorders (ED), such as anorexia and bulimia nervosa, remains essentially about $3 \%$ across the population over the years [2], affecting mostly women. These facts have led to the emergence of obesity and ED as major health concerns and to an increase in the conducted research on these fields. However, recent advances in neurobiology, genetics and sociology of eating have had minor clinical or sociological impact. In obesity, the proposed pharmacological interventions have failed [3], while the moderately successful lifestyle interventions are embedded in specific social contexts [4]. On the other hand, the more successful surgical interventions are costly, risky and associated with undesirable side effects [5]. Concerning ED, traditional interventions have poor results [6] and high levels of relapse [7], eventually degrading the patients' quality of life.

Since both obese individuals and ED patients are charac- terized by the emergence of disordered eating and abnormal physical activity, the modification of eating and physical activity behaviour have long been recognised as important factor in obesity treatment research [8]. However, eating behaviour modification has only recently drawn attention in the field of ED [9]. Based on the central assumption that obesity and ED are at the opposite extremes of the same behavioural spectrum, interventions that normalize eating patterns by training patients to eat and move in a non-pathological way have been proven to be effective in both ED and obesity in randomized control trials [10], [11]. Having proved the benefits of such interventions in patients under supervised conditions, the logical next step is to apply the same kind of methodologies to the general population by combining expertise in identifying and modifying behavioural patterns with current technical advancements in the fields of sensors, information analysis and personalised guidance systems.

SPLENDID, an EU-funded research project, has been recently initiated to undertake the aforementioned step. SPLENDID will provide personalised services guiding young individuals to adopt healthy eating behaviours and activity patterns that will prevent the onset of obesity and ED. In this context, the project has started the development of a sophisticated, noninvasive, unobtrusive, personalised, cost efficient system that can detect and normalise the behaviours putting an individual at risk for developing obesity or ED.

\section{RELATED WORK}

Early studies on eating rate changes throughout a meal have identified two distinct eating styles, namely "decelerated" (decreasing eating speed) and "linear" (constant eating speed) eating [12]. Eating behaviour recordings have indicated a higher linear to decelerated eaters ratio among both underand overweight individuals in comparison to normal-weight individuals [13]. Additionally, the linear eating style accompanied by increased or decreased food intake is suspected to be the precursor of obesity and ED, respectively [14], [15]. Furthermore, linear eaters respond to experimentally accelerated or decelerated meals very similarly to obese and ED patients, respectively [13], a fact that is clinically supported by the success of eating style normalization interventions both in ED [10] and obesity [11]. Several other behaviour patterns related to eating and daily physical activity have been identified 
as risk factors for obesity [16] and ED. Examples include increased snacking between meals, constant eating, skipping breakfast, hyperactivity or lack of physical activity. Unfortunately, most of the studies of these behaviours include suboptimal, non-objective or inherently unreliable self-reported measurements, especially for physical activity [17].

In this context, advances in sensor technology and mobile devices open new research directions towards detailed and objective behavioural monitoring for the purposes of obesity and ED prevention. With respect to eating behaviour, previous research has mainly focused on providing devices for the micro-structural description of eating. More specifically, electromyographic, oscillographic and acoustic sensor techniques have offered information about chewing [18], [19]. Technology-based monitoring of physical activity includes the use of infrared, magnetic and carbon dioxide sensors, cameras, pedometers and accelerometers. The latter are currently the preferred choice, since they are wearable/portable and can be used to quantify physical activity with high temporal resolution from raw acceleration signals [20]. For detailed dietary monitoring, very few wearable or portable sensing devices exist. Examples include intake gestures monitoring by means of accelerometers at the wrists and torso, as well as swallowing detection with the use of microphones or Surface Electromyogram (SEMG) sensors [21].

\section{Methodology}

In order to exploit the existing bidirectional relation between ED and obesity on one side and eating behaviour and human activity on the other side for the cause of prevention, SPLENDID will pursue two main lines of action.

1) By monitoring eating behaviour and activity, it will identify the risk of either developing or progressing ED or obesity.

2) By guiding people with ED or obesity to follow a desired eating behaviour and admit prescribed activity patterns, it will prevent their aggravation or even achieve improvement.

In fact, these desired eating and activity patterns are goals that are specified on a personalised and occasion dependent manner. SPLENDID aims to offer the aforementioned actions in an automatic way.

To achieve its objectives, SPLENDID will have to move the state-of-the-art forward in three distinct directions: i) It will develop and use portable, inconspicuous sensors for monitoring all aspects of behaviour relevant to obesity and ED; ii) It will develop a set of signal processing algorithms and models for automatically assessing the risk of obesity and ED, given the measured signals; iii) It will provide a methodology for measuring the proximity of individuals to prescribed goals set by a health professional (dietician, medical doctor, etc), effectively guiding them towards non-pathological behaviour and reducing their risk of obesity and ED. The SPLENDID approach is sketched in the following subsections, where the envisioned target uses and system architecture are presented.

\section{A. Target Uses}

SPLENDID will apply behavioural monitoring and modification on two distinct age groups, i.e., school-aged children

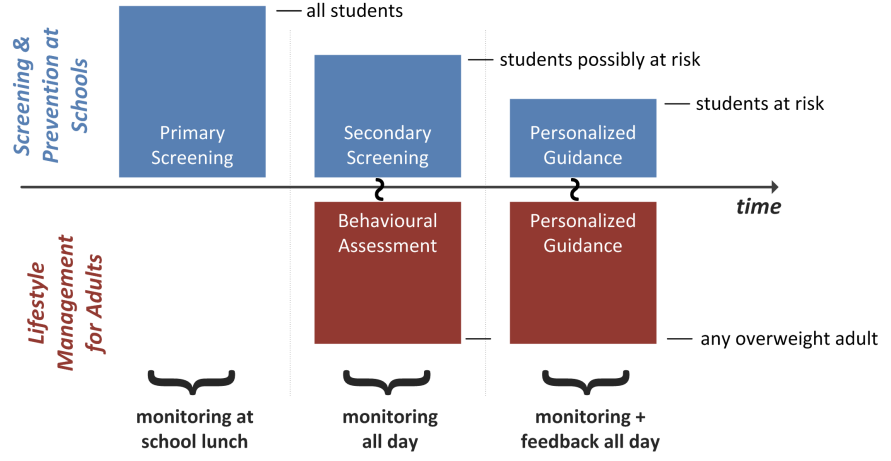

Fig. 1. Outline of the target uses of SPLENDID.

and young adults. These age groups determine two settings where SPLENDID will be employed. The corresponding target uses are described below and are visually outlined in Figure 1.

1) Screening \& Prevention Programme at Schools: The present intended use concerns the early detection and the subsequent personalised guidance of adolescents (10-17 years old children/teenagers of both genders with normal or mildly overweight BMI) at risk for developing ED or obesity. This system use is applicable to schools where students are offered daily meals and is going to be evaluated via pilot studies planned to take place at the Internationella Engelska Gymnasiet Södermalm in Stockholm (Sweden) with the support of expert health professionals. The envisioned functionality of SPLENDID for its first use is divided into three phases.

a) Primary screening: Initially, the system will record individual eating patterns during school meals for the entire school population by means of a plate with an attached scale measuring the food consumption rate during a meal. These patterns will be automatically analysed to evaluate the risk for future development of either obesity or ED. Then, external clinical experts will be provided with an automatic system report in order to confirm or contradict the quantified risk. A subset of the system users will be advised to continue to the following phase.

b) Secondary Screening: Subjects continuing from the previous phase will be monitored in their real-life and home environment to discern their extended, individualized behavioural profile that places them at risk. The acquired datasets will include: i) weight-loss-on-plate measurements during daily meals; ii) automatic sound-based swallowing and chewing quantification; iii) accelerometer-based continuous physical activity records; iv) structured, self-reported measures of fullness, physical activity and snacking between main meals. The acquired data will be evaluated by the system in real-time at the end of the monitoring period and an updated risk assessment will be generated. Same as in the first phase, external clinical experts will either confirm or contradict the system predictions. Those individuals that are definitely considered to be at risk will be asked to continue to the following phase.

c) Personalised guidance: At first, the system will set, with the help of additional input from external clinical experts, several personalized behavioural goals spanning the whole range of the measured behaviour. The same set of input sources as in the previous phase will be used in real-life and home 
environment, with the addition of feedback modules that will provide incentives for behavioural modification. The system will evaluate the compliance level to the set behavioural goals, and it will generate reports to be reviewed by the experts. The system will also provide feedback to the user, both in real-time (for direct behavioural modifications) and in discrete reports (for affecting future behaviour), as needed. The system will allow the adaptation of the set behavioural goals through supplementary expert input whenever necessary (e.g., met goals, low compliance).

2) Lifestyle Management Service for Young Adults: This intended use involves the behavioural monitoring and personalised guidance of overweight young adults (18-30 year old adults of both genders) to prevent the risk of progressing to obesity/morbid obesity. The main objective of this use is to prevent the additional weight-gain, hindering the progression of obesity and avoiding the onset of serious comorbid conditions, like type- 2 diabetes. Health expert supported pilot studies for evaluating this target use are going to be organized in Wageningen (Netherlands) by the Wageningen University. The intended system functionality for the use is divided into two phases.

a) Behavioural assessment: This phase, which is available for any overweight individual, is equivalent to the second phase of the previous system use: It utilizes the same input sources for recording the daily eating and physical activity behaviour as well as the same tools to analyse the acquired data and make predictions, which have to be verified or contradicted by external experts. Then, the experts will be able to prescribe personalised eating and activity related behavioural goals.

b) Personalised guidance: This phase - also available for any overweight individual - is directly comparable to the third phase of the previous system use. Same as before, personalized eating and activity related behavioural goals will be set with the help of input from external experts. Based on the already described input sources, feedback will be provided to the user in the form of structured reports or even in realtime and experts will be asked to confirm or contradict the predictions of the system. Re-adjustment of behavioural goals will be possible by means of expert input.

\section{B. System Architecture}

Figure 2 provides a high-level overview of the proposed architecture of SPLENDID. It comprises three main subsystems: i) the sensors; ii) the monitoring \& guidance smartphone application; iii) the decision support server.

For early detection of pathological eating behaviour, SPLENDID will use Mandometer, a scale measuring the food weight on the plate, which has been proven to be an excellent research and clinical tool for obese or ED patients [10], [13], [22]; the new generation of the device, which is developed by Mando Group AB, is equipped with Bluetooth capabilities. Another two sensors are going to be developed by the project: An Activity Meter, i.e., a triaxial accelerometer that will measure the detailed activity level of an individual throughout each day, for physical activity monitoring, and a Chewing Sensor, embedded in a standard earphone set, for detection of snacking, constant eating as well as characterisation of chewing patterns.
The measurements collected from the three sensors are transmitted via Bluetooth to the smartphone for further processing. The activity meter and the chewing sensor will share a common datalogger and Bluetooth communication device while the Mandometer will transmit data independently. The application running on an Android smartphone is responsible for i) data collection and local storage, ii) signal processing and extraction of behavioural indicators, iii) providing feedback to the end-user, and iv) aggregating the extracted indicators and the user-reported information and transmitting it to the server for processing. Examples of indicators that will be extracted by the smartphone application include the total food intake, food intake rate acceleration/deceleration, average bite size, the number and time of snacking events during the day, the activity level of the individual throughout the day, etc.

At the server-side, the extracted behavioural indicators and the user input will be aggregated and subsequently analysed for the purposes of risk assessment and personalised guidance. Risk assessment is equivalent to estimating the probability that an individual will develop obesity or ED based on her/his current status, parametrised by the extracted indicators and personal data (sex, age, current BMI, etc). Given the literature shortage in this particular domain, several approaches will need to be developed and experimentally evaluated for this task, including statistical machine learning techniques. Apart from risk assessment, the decision support subsystem will assess the proximity of the individual's behaviour to a set of goals prescribed by a health professional. Based on the evaluated proximity to goals, the system will provide feedback and recommendations to the individuals for adjusting their behaviour as needed. Finally, this subsystem will be equipped with visualization capabilities concerning all the measured and user-reported data as well as the analysis results for easy progress monitoring by both the user and the health supervisor; A web-based application is being developed for this purpose.

\section{Discussion}

SPLENDID system is currently in its design phase, where several technical and/or scientific challenges have to be overcome in order to ensure the future virtues of the system (functionality, effectiveness, user-friendliness, etc). With respect to sensors, the most important challenge concerns their ability to reliably capture the target signals - a prerequisite for accurate system predictions. Secondly, the wearable sensors must be small, inconspicuous, comfortable to wear (to ensure user-friendliness) and of low power consumption (to ensure high-portability). These criteria are indeed satisfied by the selected off-the-shelf sensor (Mandometer) and are being used as design considerations for the developed ones (Chewing Sensor and Activity Meter); Photoplythismography (PPG), bone conduction and aerial acoustic signals are the currently examined signal alternatives for the latter sensor.

Concerning the decision support algorithms, which undertake the tasks of i) indicator extraction, ii) risk assessment and iii) goal proximity evaluation, the primary challenge is accuracy. State-of-the-art techniques from the fields of signal processing (for the first task), ontology engineering (for goal expression) and machine learning (for risk classification and goal proximity evaluation) will be employed to devise robust decision support algorithms. Moreover, attention must be paid 

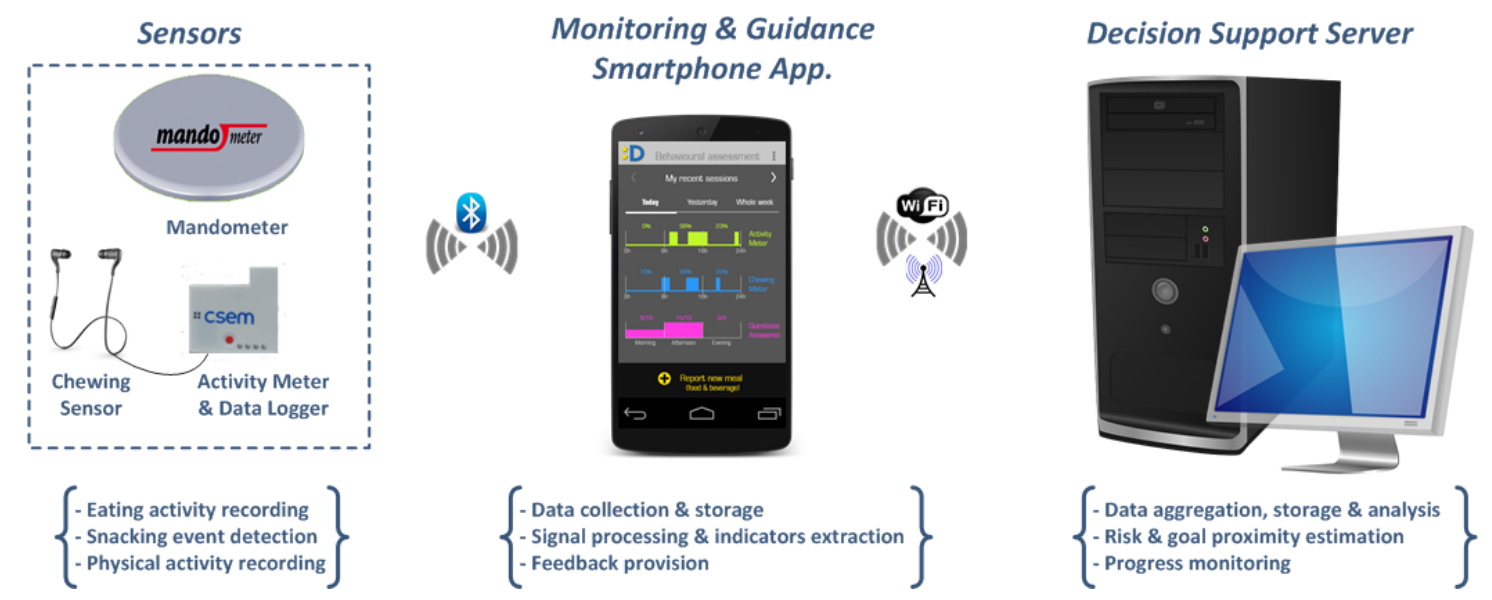

Fig. 2. High-level architecture of the SPLENDID system.

to the computational efficiency in order to allow - if possible the execution of some of these algorithms on the Smartphone or even on the micro-controllers of the employed sensors.

Finally, the user interfaces of the system are being designed with intuitiveness and attractiveness in mind to optimize the user experience and increase usage compliance. For this reason, recent developments in the fields of emotional interfaces, virtual agents and serious games have been reviewed.

\section{CONCLUSION}

The vision of SPLENDID is to design a system for obesity and ED prevention in healthy adolescents and young adults. The system will first "study" its target users in order to identify those at risk for obesity and ED; then, it will attempt to permanently modify the hazardous eating and physical activity habits of the identified individuals at risk. For this purpose, SPLENDID will design and implement an integrated system equipped with intelligent decision making capabilities, a set of commercial and novel sensors for monitoring eating and physical activity, and a couple of intuitive graphical user interfaces (a smartphone application and a web-based one) for facilitating the behaviour monitoring and modification process. The early technical challenges that are addressed during the system design phase have been briefly discussed.

\section{ACKNOWLEDGMENT}

The work leading to these results has received funding from the European Community's ICT Programme under Grant Agreement No. 610746, 01/10/2013 - 30/09/2016.

\section{REFERENCES}

[1] WHO. (2014) Who, obesity and overweight. WHO website. [Online]. Available: http://www.who.int/mediacentre/factsheets/fs311/en/

[2] S. A. Swanson et al., "Prevalence and correlates of eating disorders in adolescents: Results from the national comorbidity survey replication adolescent supplement," Archives of General Psychiatry, vol. 68, no. 7, pp. 714-723, 2011.

[3] H. Ledford, "Slim spoils for obesity drugs." Nature, vol. 468, no. 7326, pp. $878-878,2010$

[4] H. Oude Luttikhuis et al., "Interventions for treating obesity in children," Cochrane Database Syst Rev, vol. 1, no. 1, 2009.
[5] W. E. Encinosa et al., "Recent improvements in bariatric surgery outcomes," Medical care, vol. 47, no. 5, pp. 531-535, 2009.

[6] R. H. Striegel-Moore and C. M. Bulik, "Risk factors for eating disorders." American Psychologist, vol. 62, no. 3, p. 181, 2007.

[7] N. D. Berkman, K. N. Lohr, and C. M. Bulik, "Outcomes of eating disorders: a systematic review of the literature," International Journal of Eating Disorders, vol. 40, no. 4, pp. 293-309, 2007.

[8] R. J. Joseph et al., "The neurocognitive connection between physical activity and eating behaviour," obesity reviews, vol. 12, no. 10, pp. 800812, 2011.

[9] J. Treasure, V. Cardi, and C. Kan, "Eating in eating disorders," European Eating Disorders Review, vol. 20, no. 1, pp. e42-e49, 2012.

[10] C. Bergh et al., "Randomized controlled trial of a treatment for anorexia and bulimia nervosa," Proceedings of the National Academy of Sciences, vol. 99, no. 14, pp. 9486-9491, 2002.

[11] A. L. Ford et al., "Treatment of childhood obesity by retraining eating behaviour: randomised controlled trial," $B M J$, vol. 340, 2010.

[12] M. Westerterp-Plantenga et al., "The shape of the cumulative food intake curve in humans, during basic and manipulated meals," Physiol. Behav, vol. 47, no. 3, pp. 569-76, Mar 1990.

[13] I. Ioakimidis et al., "A method for the control of eating rate: a potential intervention in eating disorders," Behav Res Methods, vol. 41, no. 3, pp. 755-60, 2009.

[14] — "Description of chewing and food intake over the course of a meal," Physiol. Behav., vol. 104, no. 5, pp. 161-9, 2011.

[15] P. Södersten et al., "Obesity and the brain," Med. Hypotheses, vol. 77, no. 3, pp. 371-3, Sep 2011

[16] A. Mesas et al., "Selected eating behaviours and excess body weight: a systematic review," Obes Rev, vol. 13, no. 2, pp. 106-35, 2012.

[17] K. Pietiläinen et al., "Inaccuracies in food and physical activity diaries of obese subjects: complementary evidence from double labeled water and co-twin assessments," In J Obes (Lond), vol. 34, no. 3, pp. 437-45, Mar 2010.

[18] O. Amft, M. Kusserow, and G. Tröster, "Autoimatic identification of temporal sequences in chewing sounds," IEEE BIBM, pp. 194-201, 2007.

[19] E. Sazonov and J. Fontana, "A sensor system for automatic detection of food intake through non-invasive monitoring of chewing," IEEE Sens $J$, vol. 12, no. 5, pp. 1340-8, 2012.

[20] S. Berntsen et al., "Validity of physical activity monitors in adults participating in free-living activities," Br J Sports Med., vol. 44, no. 9, pp. 657-64, 2010.

[21] O. Amft, "Automatic dietary monitoring using on-body sensors: Detection of eating and drinking behaviour in healthy individuals," Ph.D. dissertation, Diss ETH No. 17780, 2008.

[22] C. Bergh et al., "A framework for the treatment of obesity: early support," Obesity: causes, mechanisms, preventions, and treatment. Sunderland: Sinuaer Associates, Inc, pp. 399-425, 2008. 